\title{
Uso de substâncias psicoativas entre universitários brasileiros: perfil epidemiológico, contextos de uso e limitações metodológicas dos estudos
}

\section{Use of psychoactives substances among college students: epidemiological profile, settings and methodological limitations}

\author{
Thaís Ferraz Fernandes ${ }^{1}$, Brisa Marina de Meireles Monteiro ${ }^{1}$, \\ Júlia Brighenti Menezes Silva ${ }^{1}$, Kênia Marice de Oliveira ${ }^{1}$, \\ Nájila Aélida Oliveira Viana ${ }^{1}$, Carlos Alberto Pegolo da Gama ${ }^{1}$, \\ Denise Alves Guimarães ${ }^{1}$
}

\begin{abstract}
Resumo
Introdução: O uso de substâncias psicoativas (SPAs) é reconhecido como problema de saúde pública mundial presente em diferentes segmentos populacionais, inclusive em universitários. O objetivo do estudo é analisar a produção científica nacional acerca do uso das SPAs por universitários brasileiros. Métodos: Realizou-se revisão sistemática de literatura entre 2008 e 2015 em português e inglês. Resultados: O álcool aparece como a SPA mais consumida, seguida de tabaco e maconha. O uso inicial das SPAs vincula-se à busca de diversão/prazer, e o ambiente universitário é apontado como motivador do uso. São escassos os estudos nacionais que buscam a correlação entre uso de SPAs e melhoramento cognitivo. Conclusão: Conclui-se que as pesquisas nacionais apresentam limitações ao investigar os motivos e as expectativas de uso das SPAs para além do recreativo, por exemplo, a busca por melhoria de notas ou de desempenho acadêmico.
\end{abstract}

Palavras-chave: estudantes; universidades; psicoativos.

\begin{abstract}
Introduction: The use of psychoactive substances (PAS) is recognized as a health problem, and can be observed in different populations segments, particularly among university students. This study aimed to analyze the national scientific production on the use of PAS among Brazilian college students. Methods: We performed a systematic literature review of articles published between 2008 and 2015. Results: Alcohol is the most used PAS, followed by tobacco and marijuana. At first, PASs are used for fun/pleasure, and college environment is appointed as a motivator for their use. Researches on the use of PASs and cognitive enhancement are scarce. Conclusion: National researches have limitations to study the reasons and expectations of PASs use beyond recreational use, for example, in seeking a better academic performance.

Keywords: students; university; psychotropic drugs.

1Universidade Federal de São João del-Rei (UFSJ) - Divinópolis (MG), Brasil.

Trabalho realizado na Universidade Federal de São João del-Rei (UFSJ) - Divinópolis (MG), Brasil.

Endereço para correspondência: Denise Alves Guimarães - Universidade Federal de São João del-Rei (UFSJ), Rua Sebastião Gonçalves Coelho, 400 - Chanadour - CEP: 35501-296 - Divinópolis (MG), Brasil - Email: alvesguimaraesdenise@gmail.com

Fonte de financiamento: CNPq.

Conflito de interesses: nada a declarar.
\end{abstract}




\section{INTRODUÇÃO}

Certas substâncias, por serem capazes de modificar as funções do sistema nervoso central (SNC) produzindo euforia ou alterando estados de consciência e vigília, ficaram conhecidas como substâncias psicoativas (SPAs). Alguns exemplos são a Ayahuasca, os produtos da papoula (ópio e morfina), do cânhamo (haxixe e marijuana), da fermentação de materiais orgânicos (álcool), da folha da coca (cocaína), do tabaco (nicotina) e, mais recentemente, a partir do século XIX eXX, alguns compostos sintéticos, como os barbitúricos, os benzodiazepínicos, as anfetaminas, entre outros ${ }^{1-3}$.

Ao longo da história, o homem vem utilizando e desenvolvendo diversas SPAs para variadas finalidades, como analgesia, alucinação, potencialização da memória e da concentração ${ }^{4,5}$. Comumente, 0 uso dessas substâncias é classificado de acordo com os contextos nos quais se inserem e pode ser: recreativo, que é caracterizado pelo uso de substâncias em circunstâncias sociais, com objetivos relaxantes ou em busca de prazer; laboral, que se caracteriza pela busca de alívio da carga de responsabilidade e tensões, além da melhora do desempenho no trabalho e/ou nos estudos; e/ou religioso, que visa alterar o estado de consciência, proporcionando uma melhor ligação com o sobrenatural e o divino ${ }^{1,2}$.

No entanto, atualmente, o uso de substâncias lícitas e ilícitas tem sido considerado um problema de saúde, uma vez que predispõe a acidentes, violência interpessoal, comportamentos de risco, distúrbios do sono e dependência física ou psicológica ${ }^{6}$. Além disso, o uso de SPAs tem gerado uma grande preocupação mundial devido ao número de usuários existentes e ao seu impacto sobre os indivíduos e a sociedade. O que se tem percebido é que o consumo dessas substâncias está distribuído desde estratos mais pobres até os mais ricos, abrangendo jovens, adultos e idosos. Nesse contexto e considerando-se pesquisas nacionais realizadas em populações específicas, destaca-se, com um grau de importância, o uso de SPAs entre estudantes universitários ${ }^{7}$.

O Brasil conta com mais de 7,5 milhões de estudantes universitarios, distribuídos em, aproximadamente, 2.400 instituições ${ }^{8}$. O uso de SPAs entre universitários brasileiros parece ser uma prática frequente, muito discutida pela mídia leiga ${ }^{2}$ e analisada por alguns estudos científicos. Em uma pesquisa realizada nas 27 capitais brasileiras, $49 \%$ dos 12.711 universitários participantes já haviam experimentado alguma droga ilícita pelo menos uma vez na vida $^{5}$. Além disso, 22,8\% (quase 12 milhões de pessoas) de toda a população brasileira, dos 12 aos 65 anos, já fez uso de SPAs - desconsiderando-se álcool e tabaco -, com a frequência de uso maior entre a população universitária quando comparada à população geral ${ }^{9}$. Outro estudo realizado no Estado de São Paulo observou que a frequência de uso de substâncias lícitas e ilícitas pela classe universitária no Brasil é maior do que pela população geral brasileira entre 18 e 24 anos. A prevalência do uso de álcool, por exemplo, foi de $78,6 \%$ na população geral contra $89,3 \%$ entre universitários ${ }^{10}$.

Considerando-se a relevância do tema, o presente estudo visa analisar a produção científica brasileira acerca do uso das SPAs pelos universitários.

\section{MÉTODOS}

Foi realizada uma revisão da literatura atual ${ }^{11,12}$ sobre o uso de SPAs entre estudantes universitários brasileiros. Para o levantamento bibliográfico, uma busca eletrônica foi realizada nas bases de dados Medline, Scielo, LILACS, PubMed, Science Direct, Scopus e IBECS. Os descritores utilizados foram: substâncias psicoativas AND estudantes; substâncias psicoativas AND cognição; substâncias psicoativas AND inteligência; substâncias psicoativas AND desempenho; substâncias psicoestimulantes AND desempenho; substâncias psicoativas AND universitários; substâncias psicoativas AND religião; substâncias psicoativas AND recreação; substâncias psicoativas AND recreativo; psychoactives substances AND students; psychoactives substances AND cognition; psychoactives substances AND inteligence; psychoactives substances AND performance; psychoactives substances AND university; psychoactives substances AND college students; psychostimulants AND performance; psychoactives substances AND religion; psychoactives substances AND recreational. Esses termos foram aplicados em todas as bases de dados utilizadas.

Foram incluídos na seleção artigos originais em português e em inglês, publicados entre os anos de 2008 a 2017. Não foram incluídos: (i) estudos realizados com estudantes não universitários; (ii) estudos realizados com plantas e animais; (iii) estudos laboratoriais com análise de substâncias biológicas; (iv) estudos realizados fora do Brasil; (v) artigos não originais; (vi) artigos duplicados. Para seleção dos artigos, primeiramente foi feita uma análise por título e, posteriormente, por resumo, o que permitiu a exclusão daqueles que extrapolavam os objetivos deste estudo. Os artigos selecionados foram lidos integralmente e só então, uma vez atendidos aos critérios, incluídos nesta revisão. Os estudos foram avaliados por dois autores, de forma independente em relação à elegibilidade (obtendo-se uma convergência entre os resultados encontrados por eles em $90 \%$ ), e, em caso de discordância, um terceiro avaliador foi solicitado. Os dados foram extraídos e dispostos em um formulário padrão, adaptado da Colaboração Cochrane ${ }^{13,14}$. A partir da apreciação dos conteúdos de todas as referências escolhidas e de acordo com os referenciais do método de análise de conteúdo ${ }^{15,16}$, emergiram as seguintes categorias: 1 ) desenhos de pesquisa e instrumentos utilizados; 2) regiões do Brasil e instituições pesquisadas; 3 ) tipo e prevalência de uso de SPAs; 4) faixa etária, período/frequência de uso e diferenças por sexo; 5) motivações, fatores protetores e fatores de risco.

\section{RESULTADOS}

A estratégia de busca eletrônica retornou 4.853 estudos, dos quais 4.816 foram excluídos após a leitura dos títulos e resumos. Portanto, 37 estudos foram selecionados para a leitura completa do texto, sendo oito excluídos nessa etapa. Dessa forma, o número total de estudos incluídos e descritos no presente estudo foi de 29 (Figura 1 e Tabela 1). 
Tabela 1. Estudos originais

\begin{tabular}{|c|c|c|c|c|}
\hline Autores & Local e ano & Tamanho amostral/curso e faixa etária & $\begin{array}{l}\text { Desenho de } \\
\text { estudo }\end{array}$ & Instrumento de pesquisa \\
\hline Colares et al. ${ }^{17}$ & Pernambuco, 2009 & $\begin{array}{l}\text { - } 382 \text { estudantes do final de cursos da área da } \\
\text { saúde } \\
\text { - Faixa etária de } 20 \text { a } 29 \text { anos }\end{array}$ & Quantitativo & Questionário estruturado \\
\hline Oliveira et al. ${ }^{18}$ & Santo André/SP, 2009 & $\begin{array}{l}\text { - } 274 \text { estudantes do } 2^{\circ} \text { e } 3^{\circ} \text { ano de Medicina e } \\
\text { Enfermagem } \\
\text { - Faixa etária entre } 18 \text { e } 24 \text { anos }\end{array}$ & Quantitativo & Questionário estruturado \\
\hline Bortoluzzi et al. ${ }^{19}$ & Meio-Oeste/SC, 2012 & $\begin{array}{l}\text { - } 384 \text { estudantes } \\
\text { - Faixa etária entre } 18 \text { e } 52 \text { anos }\end{array}$ & Quantitativo & Questionário estruturado \\
\hline Medeiros et al..$^{20}$ & Gravataí/RS, 2012 & $\begin{array}{l}\text { - } 560 \text { estudantes } \\
\text { - Faixa etária entre } 17 \text { e } 74 \text { anos }\end{array}$ & Quantitativo & Questionários estruturados \\
\hline Santos et al. ${ }^{21}$ & Vitória/ES, 2013 & $\begin{array}{l}\text { - } 221 \text { alunos do curso de Psicologia } \\
\text { - Faixa etária de } 18 \text { a } 24 \text { anos }\end{array}$ & Quantitativo & Questionário estruturado \\
\hline Portugal et al..$^{22}$ & Vitória/ES, 2014 & $\begin{array}{l}\text { - } 215 \text { estudantes do curso de Pedagogia } \\
\text { - Faixa etária de } 18 \text { a } 24 \text { anos }\end{array}$ & Quantitativo & Questionário estruturado \\
\hline Zalaf et al. ${ }^{23}$ & São Paulo/SP, 2009 & $\begin{array}{l}\text { - } 8 \text { participantes, moradores ou hóspedes } \\
\text { regulares dos blocos de moradia da USP } \\
\text { - Faixa etária não especificada }\end{array}$ & Qualitativo & Entrevista semiestruturada \\
\hline Gomes et al. ${ }^{24}$ & 27 capitais do Brasil, 2009 & $\begin{array}{l}\text { - } 12.595 \text { estudantes universitários de cursos } \\
\text { de graduação presencial, de IES públicas e } \\
\text { privadas } \\
\text { - Faixa etária não especificada }\end{array}$ & Quantitativo & Questionário estruturado \\
\hline Maia et al. ${ }^{25}$ & Teresina/PI, 2017 & $\begin{array}{l}\text { - } 291 \text { estudantes de Medicina } \\
\left(1^{\circ} \text { ao } 8^{\circ} \text { período }\right) \\
\text { - Faixa etária não especificada }\end{array}$ & Quantitativo & Questionário estruturado \\
\hline Tostes et al. ${ }^{26}$ & Itajubá/MG, 2016 & $\begin{array}{l}\text { - } 419 \text { alunos do curso de Medicina do } \\
1^{\circ} \text { ao } 6^{\circ} \text { ano } \\
\text { - Maiores de } 18 \text { anos (média de } 22 \text { anos) }\end{array}$ & Quantitativo & Questionário estruturado \\
\hline Silva e Tucci ${ }^{27}$ & Diadema/SP, 2016 & $\begin{array}{l}\text { - } 407 \text { estudantes (Ciências Ambientais, } \\
\text { Ciências Biológicas, Farmácia, Bioquímica, } \\
\text { Química Industrial, Química, Engenharia e } \\
\text { Ciências) } \\
\text { - Média de idade de } 21,86 \text { anos }\end{array}$ & Quantitativo & Questionários estruturados \\
\hline Botti et al. ${ }^{28}$ & Divinópolis/MG, 2016 & $\begin{array}{l}\text { - } 179 \text { estudantes de Enfermagem } \\
\text { - Média de idade de } 26 \text { anos }\end{array}$ & Quantitativo & Questionários estruturados \\
\hline Silva et al. ${ }^{29}$ & Estado do Acre, 2016 & $\begin{array}{l}\text { - } 12 \text { alunos do } 6^{\circ} \text { período de Enfermagem } \\
\text { - Faixa etária entre } 19 \text { e } 35 \text { anos } \\
\text { (média de } 24 \text { anos) }\end{array}$ & Qualitativo & Entrevista semiestruturada \\
\hline Veiga et al. ${ }^{30}$ & Itararé/SP, 2016 & $\begin{array}{l}\text { - } 69 \text { alunos do } 5^{\circ} \text { e } 6^{\circ} \text { semestre do curso de } \\
\text { licenciatura em Educação Física } \\
\text { - Média de idade de } 21 \text { anos }\end{array}$ & Quantitativo & Questionários estruturados \\
\hline Pimentel et al. ${ }^{31}$ & João Pessoa/PB, 2009 & $\begin{array}{l}\text { - } 276 \text { estudantes dos cursos de Administração, } \\
\text { Ciências Sociais, Computação, Direito, } \\
\text { Ciências Contábeis e Psicologia } \\
\text { - Faixa etária entre } 17 \text { e } 43 \text { anos }\end{array}$ & Quantitativo & Questionários estruturados \\
\hline Lemos et al. ${ }^{32}$ & Salvador/BA, 2007 & $\begin{array}{l}\text { - } 404 \text { estudantes de Medicina } \\
\text { - Média de idade de } 22 \text { anos }\end{array}$ & Quantitativo & Questionário estruturado \\
\hline Carvalho et al..$^{33}$ & $\begin{array}{l}\text { Interior do Estado de São } \\
\text { Paulo, } 2009\end{array}$ & $\begin{array}{l}\text { - } 325 \text { estudantes dos cursos de Enfermagem, } \\
\text { Farmácia e Odontologia das } 2^{\circ} \text { e } 3^{\circ} \text { ano- } \\
\text { Faixa etária entre } 18 \text { e } 24 \text { anos }\end{array}$ & Quantitativo & Questionário estruturado \\
\hline Botti et al. ${ }^{34}$ & Betim/MG, 2010 & $\begin{array}{l}\text { - } 393 \text { estudantes do curso de Enfermagem } \\
\text { - Faixa etária não especificada } \\
\end{array}$ & Quantitativo & Questionário estruturado \\
\hline Picolotto et al. ${ }^{35}$ & Passo Fundo/RS, 2010 & $\begin{array}{l}\text { - } 266 \text { alunos do curso de Enfermagem } \\
\text { - Faixa etária de } 23 \text { a } 36 \text { anos } \\
\end{array}$ & Quantitativo & Questionário estruturado \\
\hline Teixeira et al. ${ }^{36}$ & Espírito Santo, 2010 & $\begin{array}{l}\text { - } 174 \text { estudantes do curso de Odontologia } \\
\text { - Faixa etária: maioria entre } 20 \text { a } 22 \text { anos }\end{array}$ & Quantitativo & Questionário estruturado \\
\hline Amorim et al. ${ }^{37}$ & João Pessoa/PB, 2012 & $\begin{array}{l}\text { - Estudantes de Medicina do } 1^{\circ} \text { ao } 5^{\circ} \text { ano } \\
\text { - Faixa etária não especificada }\end{array}$ & Quantitativo & Questionários estruturados \\
\hline
\end{tabular}


Tabela 1. Continuação..

\begin{tabular}{|c|c|c|c|c|}
\hline Autores & Local e ano & Tamanho amostral/curso e faixa etária & $\begin{array}{l}\text { Desenho de } \\
\text { estudo }\end{array}$ & Instrumento de pesquisa \\
\hline Carneiro et al. ${ }^{38}$ & Volta Redonda/RJ, 2011 & $\begin{array}{l}\text { - } 156 \text { alunos do } 1^{\circ} \text { ao } 8^{\circ} \text { período do curso de } \\
\text { Medicina } \\
\text { - Faixa etária não especificada }\end{array}$ & Quantitativo & Questionário estruturado \\
\hline Pillon et al. ${ }^{39}$ & Ribeirão Preto/SP, 2005 & $\begin{array}{l}\text { - } 200 \text { estudantes de } 1^{\circ} \text { ano de graduação das } \\
\text { áreas de Biológicas, Exatas e Humanas } \\
\text { - Faixa etária entre } 18 \text { e } 26 \text { anos }\end{array}$ & Quantitativo & Questionário estruturado \\
\hline Morgan et al. ${ }^{40}$ & Rio Grande/RS, 2017 & $\begin{array}{l}\text { - } 200 \text { alunos do } 1^{\circ} \text { ao } 4^{\circ} \text { ano do curso de } \\
\text { Medicina } \\
\text { - Idade média de } 23,3 \text { anos }\end{array}$ & Quantitativo & Questionário estruturado \\
\hline Dázio et al. ${ }^{41}$ & Alfenas/MG, 2015 & $\begin{array}{l}\text { - } 20 \text { estudantes de graduação das áreas de } \\
\text { Ciências da Saúde, Ciências Humanas e } \\
\text { Letras e Exatas } \\
\text { - Faixa etária de } 20 \text { a } 32 \text { anos }\end{array}$ & $\begin{array}{l}\text { Estudo } \\
\text { etnográfico }\end{array}$ & $\begin{array}{l}\text { Questionário estruturado } \\
\text { mais entrevista } \\
\text { semiestruturada }\end{array}$ \\
\hline Santos et al. ${ }^{42}$ & Cuiabá/MT, 2017 & $\begin{array}{l}\text { - } 637 \text { estudantes de diversos cursos } \\
\text { - Faixa etária não especificada } \\
\end{array}$ & Quantitativo & Questionário estruturado \\
\hline Damasceno et al. ${ }^{43}$ & Juquié/BA, 2016 & $\begin{array}{l}\text { - } 97 \text { universitários do curso de Educação Física } \\
\text { - Faixa etária de } 18 \text { a } 32 \text { anos }\end{array}$ & Quantitativo & Questionários estruturados \\
\hline Ferraz et al. ${ }^{44}$ & Sul do Brasil, 2017 & $\begin{array}{l}\text { - } 284 \text { acadêmicos dos cursos de Medicina, } \\
\text { Direito e Engenharia Civil } \\
\text { - Média de idade de } 23 \text { anos }\end{array}$ & Quantitativo & Questionário estruturado \\
\hline Petroianu et al. ${ }^{45}$ & Belo Horizonte/MG, 2010 & $\begin{array}{l}\text { - } 353 \text { estudantes de todos os anos do curso de } \\
\text { Medicina } \\
\text { - Faixa etária de } 18 \text { a } 41 \text { anos }\end{array}$ & Quantitativo & Questionário estruturado \\
\hline
\end{tabular}

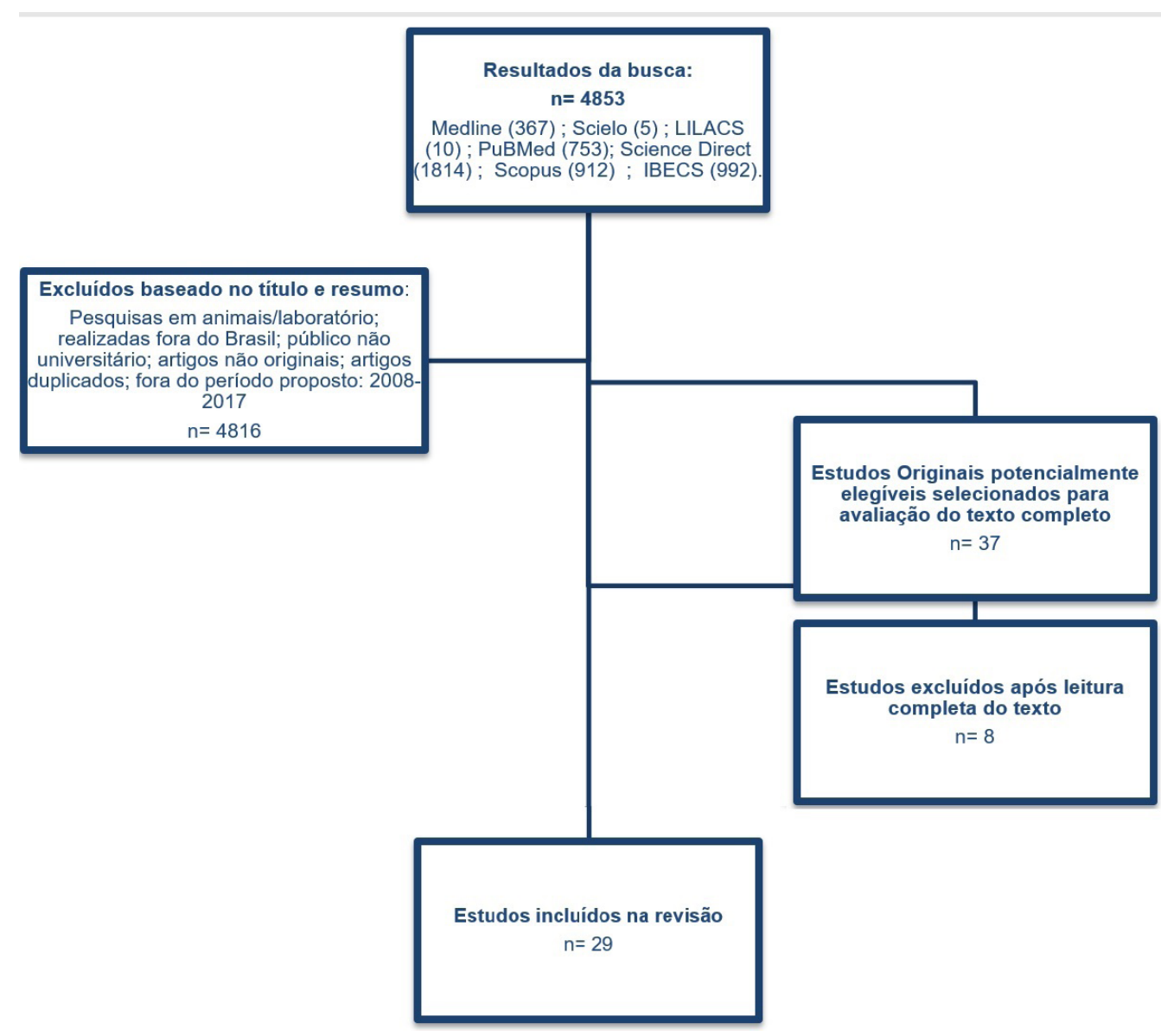

Figura 1. Fluxograma de inclusão e exclusão dos estudos 


\section{Desenhos de pesquisa e instrumentos utilizados}

Dos 29 artigos analisados, 27 apresentavam como instrumento de pesquisa os questionários fechados, e dois, entrevistas semiestruturadas. Desses 27 que foram construídos a partir de questionários fechados, nove incluíam uma questão com opção aberta de resposta. Ainda, 27 estudos usaram questionários elaborados para a própria pesquisa ou adaptados dos originais, com predomínio de questões fechadas. Por fim, 26 utilizavam questionários autoaplicados, e nove, mais de um instrumento na investigação ${ }^{18,27,28,31,33,34,37,42,43}$.

Os instrumentos de pesquisa utilizados em sua versão original ou adaptada foram: o questionário da Organização Mundial de Saúde (OMS) (1980) 25,27,28,32,34,36,42,43,45 , I Levantamento Nacional sobre o Uso de Álcool, Tabaco e Outras Drogas entre Universitários das 27 Capitais Brasileiras ${ }^{20,22,24}$, National College Health Risk Behavior Survey (NCHRBS) ${ }^{17}$, Core Survey (1989) ${ }^{18,33}$, Canadian Survey, n.d ${ }^{18}$, Centro Brasileiro de Informações sobre Drogas Psicotrópicas (CEBRID) ${ }^{19,35}$, Alcohol Smoking and Substance Involvement Screening Test (ASSIST) $)^{5,6,22,28,30}$, Alcohol Use Disorders Identification Test (AUDIT) $)^{25,27,28,37,43}$, Beck Depression Inventory (BDI), Beck Scale for Suicide Ideation (BSI ${ }^{34}$, WHOQOL-Bref ${ }^{28}$, Escala de Atitudes Frente ao Uso de Álcool (EAFUA), Escala de Atitudes Frente ao Uso de Maconha (EAFUM), Escala de Atitudes Frente ao Uso de Drogas (EAFUD) $)^{31}$, Fagerström Tolerance Questionnaire ${ }^{37}$, International Physical Activity Questionnaire (IPAQ - short version) $)^{37}$, Youth Risk Behavior Survey (YRBS) ${ }^{39}$ e The Smart Drug Study ${ }^{40}$.

Os modelos de questionário mais utilizados nas pesquisas, seja na versão original, seja na versão adaptada, foram o da OMS (1980) em oito pesquisas s, $28,32,34,36,42,43,45$ o o AUDIT ${ }^{25,27,28,37,43} \mathrm{em}$ cinco pesquisas.

O questionário construído pela OMS (1980) - versão adaptada - é de autopreenchimento, composto por oito questões fechadas, das quais apenas a primeira questão, que investiga o tipo de substância utilizada, apresenta a opção aberta de resposta sobre o uso de outras substâncias além das descritas, que são: tabaco (cigarros, charutos, cigarrilhas etc.), álcool (cervejas, vinhos, licores, bebidas espirituosas, shots etc.), cannabis (haxixe, erva, marijuana, pólen etc.), cocaína (coca, crack etc.), estimulantes de tipo anfetamina (speed, anfetaminas, ecstasy etc.), inalantes (cola, gasolina, óxido nitroso, solvente etc.), ansiolíticos, sedativos, hipnóticos, alucinogénios (LSD, cogumelos, PCP, ketamina etc.), opiáceos (heroína, morfina, metadona, buprenorfina, codeína etc.). O questionário aborda, ainda: frequência de uso; se houve desejo de utilizar nos últimos três meses; se o uso resultou em algum problema de saúde, social, legal ou financeiro; se o participante deixou de fazer alguma atividade que era normalmente esperada; se alguma pessoa próxima se mostrou preocupada com o seu uso; se houve desejo de controlar, diminuir ou interromper o uso e não conseguiu; e se já houve uso de drogas injetáveis.
O segundo instrumento mais utilizado foi o AUDIT, que é um questionário composto por dez questões objetivas, elaborado pela OMS para o rastreio e a intervenção no consumo de risco ou uso nocivo de álcool, e recomendado pela Direção Geral de Saúde (DGS). É também de autopreenchimento e está estruturado em blocos temáticos: as três primeiras perguntas correspondem ao AUDIT-C (consumption) e visam detectar o consumo de risco; as perguntas quatro a seis correspondem aos sintomas de dependência; as perguntas sete a dez tratam dos sintomas de consumo nocivo, que, segundo a OMS, é o padrão de consumo que causa danos à saúde - físicos ou mentais - e que não satisfaz aos critérios de dependência.

Apenas duas pesquisas ${ }^{38,40}$ investigavam a relação entre o uso de SPAs e a busca de melhoramento cognitivo, mais especificamente considerando o uso de metilfenidato entre os estudantes: na primeira $^{40}$, buscou-se encontrar efeitos adversos da medicação percebidos pelos participantes; na segunda ${ }^{38}$, investigou-se o uso de metilfenidato para melhorar o desempenho cognitivo.

Somente dois estudos utilizavam pesquisas com entrevistas semiestruturadas ${ }^{39}$, com roteiros construídos especialmente para a pesquisa e cujos principais temas foram: o uso problemático de álcool e outras drogas em uma moradia estudantil ${ }^{39}$ e os significados que o estudante atribui ao ambiente familiar com ênfase nos fatores que influenciam positiva e negativamente o consumo de álcool e outras drogas ${ }^{29}$. Além disso, dos estudos analisados, 26 eram pesquisas quantitativas, duas, qualitativas ${ }^{29,39}$, e uma, mista (quanti-quali) ${ }^{41}$.

\section{Regiões do Brasil e instituições pesquisadas}

Dos artigos analisados, 16 pesquisas foram conduzidas com estudantes da região Sudeste $18,20,22-24,26-28,30,33,34,36,38,39,41,45$, sete, da região Nordeste ${ }^{17,24,25,31,32,37,43}$, seis, da região Sul ${ }^{19,20,24,35,40,44}$, dois, da região Norte ${ }^{24,29}$, dois, da região Centro-Oeste ${ }^{24,42}$, e apenas um estudo abrangeu todas as regiões do país ${ }^{24}$. Dos 26 artigos que identificaram a cidade de realização da pesquisa, nove foram realizadas nas capitais brasileiras ${ }^{21-25,31,32,37,45}$. Das pesquisas analisadas, 11 ocorreram em instituições públicas ${ }^{17,23,24,27,31,33,34,42,43,45}$, três, em instituições particulares ${ }^{26,31,34}$, e as demais não mencionaram o tipo de instituição em que a pesquisa foi realizada. Três estudos apontaram a área de atuação ${ }^{17,39,41}$, e 20, o curso em que os alunos estavam matriculados ${ }^{18,21,24-30,32-38,40,43-45}$. Apenas um estudo ${ }^{44} \mathrm{dos}$ artigos analisados comparou diferentes áreas de atuação dentro do mesmo ambiente universitário em relação ao consumo de álcool e outras drogas. Essa população era composta por acadêmicos de um curso da área de saúde, um de humanas e um de exatas. Considerando as áreas de cursos mencionadas, destacou-se a área da saúde com 23 estudos, e os principais cursos representados foram Medicina (2.400 estudantes) e Enfermagem (1.175 estudantes) ${ }^{18,25,26,28,29,32-35,37,38,40,44,45}$. 


\section{Tipo e prevalência de uso de substâncias psicoativas}

Do conjunto de instrumentos analisados, há uma variação dos tipos de substância investigada. Dos 29 artigos analisados, 15 deles $^{18-22,26,27,30-34,36,37,45}$ avaliaram quantitativamente o tipo de substância utilizada, e as SPAs mais consumidas entre os universitários foram o álcool e o tabaco. O álcool foi a SPA de maior prevalência, sendo citado em 27 dos 29 artigos analisados. Em segundo lugar, apareceu o tabaco, mencionado em 13 artigos $^{17-22,24,31-34,36,45}$. Alguns estudos apontaram que a grande parte dos fumantes também consumia bebidas alcoólicas $^{17-22,35,36,45}$. Em terceiro lugar, apareceu a maconha como a SPA mais consumida de maneira geral e como a droga ilícita mais utilizada pelos universitários ${ }^{18-21,31,33,34}$.

Apenas dois estudos ${ }^{38,40}$ investigaram substâncias que não incluíam o álcool, nos quais foram abordados o uso de psicoestimulantes por estudantes universitários, como o metilfenidato.

\section{Faixa etária, período/frequência de uso e diferenças por sexo}

Dos 29 artigos analisados, a maioria especificou a faixa etária dos participantes ${ }^{14,17-22,29,33,35,36,39,42,45}$, outros identificaram a média de idade ${ }^{26-28,30,32,40,43} \mathrm{e}$ os demais não abordaram a idade pesquisada ${ }^{23-25,34,37,38,41}$. Dos estudos que não identificaram a faixa etária ou a média de idade dos participantes, alguns apontaram os períodos dos cursos pesquisados ${ }^{25,37,38}$. Para aqueles que especificaram a faixa etária, é possível observar que a mais prevalente foi entre 18 e $24 \operatorname{anos}^{18,21,22,33}$. Entre aqueles que citaram a média de idade dos participantes, ela variou entre 21 e 26 anos.

Do total de 29 artigos analisados, 13 utilizaram alguma forma de avaliação em relação ao período/frequência do uso e outros 16 não fizeram essa avaliação.

Dos estudos que avaliaram período/frequência de uso, é possível observar uma variedade de intervalos temporais utilizados para fazer essa mensuração. Alguns estudos ${ }^{18,33}$ usaram intervalos anuais, mensais, semanais e diários e identificaram como principais SPAs consumidas nesses intervalos o álcool e a maconha. Outro estudo ${ }^{20}$, que utilizou intervalos temporais vinculados ao uso nos três últimos meses, identificou como principais substâncias utilizadas nas categorias o álcool, seguido do tabaco, da maconha e das anfetaminas ou do ecstasy.

Uma pesquisa ${ }^{25}$, que avaliou somente o consumo de álcool, utilizou intervalos temporais semanais e identificou que a bebida mais consumida nas categorias "maior ou igual a cinco vezes/semana" e "uma a quatro vezes/semana" foi a cerveja e a mais consumida na categoria "menor que uma vez/semana" foi o vinho. Outra pesquisa ${ }^{44}$, que avaliou a frequência do uso de álcool e tabaco, considerando intervalos temporais anuais, mensais, semanais e diários, identificou que o álcool foi a SPA mais utilizada nas categorias "uma ou duas vezes ao ano", "mensalmente", "semanalmente", "diariamente ou quase todos os dias".

Os outros oito estudos ${ }^{19,21,22,26,32,34,36,39}$ avaliaram a frequência do uso de SPAs considerando como intervalos temporais o "uso na vida", "uso no ano" e "uso no mês". Esses três intervalos temporais foram empregados em conjunto por alguns estudos ${ }^{19,21,22,32,36}$ que identificaram o álcool como a SPA mais utilizada. Em segundo lugar, apareceu o tabaco ${ }^{19,21}$ para as três categorias, os tranquilizantes 22 para "uso no ano" e "uso no mês" e o lança-perfume ${ }^{32}$ para "uso na vida" e "uso no ano". O tabaco apareceu também em segundo lugar para "uso na vida" e "uso no mês"

Das 13 pesquisas que avaliaram período/frequência de uso, apenas duas ${ }^{27,43}$ abordaram a classificação de risco para uso das SPAs, por meio do questionário AUDIT. Esses dois estudos concluíram que a maioria dos participantes foi classificada como em uso de baixo risco. Os outros três estudos ${ }^{25,28,37}$ que utilizaram esse mesmo instrumento de pesquisa não abordaram o perfil de risco de consumo das SPAs.

Dos 29 artigos analisados, quatro abordaram a idade média de iniciação do uso de álcool, que variou de 13 a 16,01 anos a $^{21,22,32,39}$, e três, a idade média de iniciação do uso de tabaco, que variou de 15,71 a 16,55 anos $^{21,22,32}$. Além deles, um estudo ${ }^{34}$ verificou que as mulheres apresentaram prática de uso de bebida alcoólica, tabaco, ansiolíticos, anorexígenos e maconha mais precoce do que os homens, enquanto os homens apresentaram prática mais precoce de uso de inalantes quando comparados às mulheres.

Em relação aos artigos que trataram dos períodos de maior consumo das substâncias na faculdade ${ }^{26,45}$, um deles concluiu que $73 \%$ dos alunos referiram aumento no consumo de bebidas alcoólicas após ingressar na faculdade ${ }^{26}$; já o outro concluiu que estimulantes eram utilizados principalmente nos últimos anos do curso médico ${ }^{45}$.

À exceção de um estudo ${ }^{41}$, todos os outros incluíram homens e mulheres como participantes de pesquisa. Além disso, dos 29 artigos analisados, 17 deles $^{18-22,25-27,29,33-37,40,44,45}$ apresentaram predomínio do sexo feminino na amostra geral, dois tiveram amostra masculina maior ${ }^{32,42}$ e apenas um possuía a mesma quantidade entre homens e mulheres ${ }^{39}$.

Dos 28 artigos que consideraram homens e mulheres na composição amostral, 13 deles discutiram as diferenças de consumo das substâncias pesquisadas entre os sexos. Em relação à frequência de uso de bebida alcoólica entre homens e mulheres, apenas três estudos concluíram que não houve diferença estatisticamente significativa no consumo "na vida" de álcool ${ }^{36,44,45}$. Sete estudos apontaram que o uso de álcool foi mais frequente entre os estudantes do sexo masculino ${ }^{17,26,32,35,38,39,43}$, à exceção de uma pesquisa que alegou que houve predomínio do uso "na vida" de bebida alcoólica por estudantes do sexo feminino em relação ao masculino ${ }^{34}$. 
No que se refere à frequência de uso de tabaco entre homens e mulheres, todos os estudos que abordaram o uso dessa substância concluíram que o consumo foi maior entre os homens quando comparados às mulheres ${ }^{17,32,34,36,44}$.

Quanto à frequência de uso de maconha entre os universitários, quatro pesquisas concluíram que o consumo foi maior entre os homens quando comparados às mulheres ${ }^{17,24,32,34}$.

\section{Motivações, fatores protetores e fatores de risco}

Dos 29 artigos analisados, oito investigaram os motivos apontados pelos universitários para o uso de SPAs ${ }^{20,23,32,38-41,45}$. Para aqueles que analisaram as motivações para o uso geral de SPAs, os principais motivos alegados foram diversão ${ }^{20,32,39}$, fuga da realidade ${ }^{23}$, válvula de escape ${ }^{41}$ e potencialização das atividades acadêmicas ${ }^{20,45}$, sendo esta última motivação compatível com o que se considera como uso laboral ${ }^{1,2}$. Apenas duas pesquisas investigaram as motivações do consumo específico para estimulantes (cafeína, energético, anfetamina, metilfenidato, ecstasy), e os motivos alegados foram: permanecer desperto, compensar a privação do sono e melhorar raciocínio, atenção e memória ${ }^{38,40}$.

Ainda, das 29 pesquisas, sete analisaram fatores protetivos relacionados ao uso de $\mathrm{SPAs}^{21,22,24,27,29,35,45}$, dos quais: dois apresentaram a religião como fator de proteção para o consumo de drogas em geral ${ }^{22,24}$; um como fator de proteção para o consumo de álcool ${ }^{27}$; um como fator de proteção para o consumo de tabaco ${ }^{21}$; e um como fator de proteção para o uso de benzodiazepínicos ${ }^{35}$. Além disso, dois artigos abordaram o fato de morar com a família e de exercer uma profissão como fatores protetivos para uso de SPAs em geral ${ }^{29,45}$.

Dos 29 artigos analisados, dez pesquisaram fatores de risco e vulnerabilidade relacionados ao uso de SPAs ${ }^{14,20-23,25,29,30,35,45}$, dos quais: quatro abordaram o consumo de SPAs como facilitadores de uso para outras substâncias ${ }^{14,21,26,45}$; dois concluíram que a moradia estudantil (distante da família) era considerada um fator facilitador do uso ${ }^{20,23}$; um abordou o exemplo familiar e a postura permissiva dos pais como fator de predisposição ao uso ${ }^{29}$; e dois trataram da classe econômica elevada como fator de risco para o uso de substâncias ilícitas ${ }^{22,35}$. Além disso, um estudo concluiu que o sexo masculino, por si só, é considerado um fator de risco para o consumo de SPA ${ }^{25}$.

\section{DISCUSSÕES}

A maior parte dos instrumentos analisados apresentou um perfil semelhante: focado em levantamentos epidemiológicos, com apresentação de resultados quantitativos - em números percentuais na maioria das vezes. Nesses instrumentos de pesquisa, as questões eram referentes a: dados sociodemográficos; curso e período em que os participantes estavam matriculados; tipos de SPAs utilizadas com basicamente as mesmas opções de respostas (álcool, tabaco, maconha, lança-perfume, cocaína, tranquilizantes, anfetaminas). Poucas pesquisas foram desenvolvidas com instrumentos de pesquisa que continham questões totalmente abertas, permitindo que o participante declarasse livremente quais substâncias já utilizou e quais eram as motivações, dando respostas que iam além de opções previamente determinadas. No entanto, pela diversidade de métodos, desenhos de pesquisa e amostras dos estudos, a possibilidade de estabelecer comparações precisas entre todos os resultados apresentados nas investigações é limitada.

As discussões sobre "motivações para o uso de SPA" estão prioritariamente voltadas para uma categoria mais comumente encontrada nas pesquisas brasileiras: o uso recreativo. Ressalta-se que esses resultados podem refletir um fator cultural e estar relacionados a limitações metodológicas, uma vez que os instrumentos de coleta se baseiam com frequência em questionários predominantemente fechados, com categorias já consagradas na literatura e que não abordam, ou o fazem de forma insuficiente, novas possibilidades de uso de SPAs na atualidade, por exemplo, a relação entre uso de SPAs e busca de melhoramento cognitivo ou alívio de tensões geradas pelo ambiente acadêmico.

De acordo com o censo realizado em 2011, o Brasil contava com 2.365 Instituições de Ensino Superior (IES), das quais 284 públicas e 2.081 privadas. Quando se considera a distribuição das IES por região brasileira, percebe-se maior concentração no Sudeste $(49 \%=1.157)$ e menor no Norte $(6 \%=152)^{46}$. A concentração das pesquisas na região Sudeste parece refletir as divergências em relação ao desenvolvimento econômico e às consequentes diferenças de financiamento e de infraestrutura relacionadas ao desenvolvimento de ciência nas diferentes regiões do país. Além disso, não se pode desconsiderar que a concentração de IES na região Sudeste está diretamente relacionada a um maior número de docentes e pesquisadores interessados em pesquisas cujo público-alvo são os universitários.

O álcool foi a SPA mais utilizada entre os universitários brasileiros, seguido do tabaco. Em terceiro lugar, apareceu a maconha como a mais utilizada no geral, mas, entre as substâncias ilícitas, apareceu em primeiro lugar. Esses dados corroboram os resultados encontrados em estudos internacionais.

Constata-se, na sociedade atual, uma naturalização do consumo de álcool e tabaco, pelo fato de serem SPAs lícitas. No entanto, destaca-se que, em termos de carga global das doenças, a maior parte dos problemas está ligada ao consumo de substâncias lícitas, e não ao consumo de substâncias ilícitas ${ }^{47}$.

A composição das amostras das populações pesquisadas mostrou a predominância de jovens e a grande participação de mulheres nessas pesquisas, o que pode apontar o aumento do acesso delas à formação universitária. No entanto, pouco menos da metade das pesquisas se ocupou em analisar diferenças de consumo para os sexos. De forma geral, homens e mulheres das amostras pesquisadas fizeram uso de SPAs, especialmente álcool e tabaco. 
Os principais motivos declarados nas pesquisas para o uso de SPA por universitários brasileiros foram os recreativos (diversão, válvula de escape e fuga da realidade). Em segundo lugar, apareceram os motivos laborais, relacionados às tentativas de aliviar condições estressantes e potencializar a capacidade de aprendizado, atenção e memória. O uso de SPA relacionada à potenciação cognitiva começou a aparecer nos estudos nacionais mais recentes, acompanhando a tendência de estudos internacionais que têm se dedicado a essa investigação $0^{48,49}$.

Conforme apontado nas pesquisas, as motivações para o consumo de SPAs por estudantes universitários são bastante amplas e de análise complexa, podendo estar relacionadas a aspectos externos, como a família, os meios de comunicação e os amigos, ou a aspectos internos, como as características pessoais, a necessidade de pertença, a curiosidade, o prazer e a ociosidade ${ }^{34}$.

\section{CONCLUSÕES}

A vida universitária é um período que pode possibilitar experiências novas e a conquista de uma profissão, bem como pode se configurar como um período crítico, de maior vulnerabilidade para o início e a manutenção do uso das SPAs.
O uso de SPAs por estudantes universitários é tema recorrente de diversos estudos nacionais e internacionais, visto que se trata de um assunto relevante e capaz de impactar a vida acadêmica e pessoal do indivíduo. Deve-se ter maior cuidado e atenção àquelas ditas lícitas, pois têm um aparato jurídico que protege sua fabricação e seu consumo e são comercializadas sem restrições de horário ou estabelecimento comercial. Essa facilidade de acesso e de consumo justifica a elevada prevalência do uso de álcool, seguido pelo tabaco, encontrado em todos os estudos, além de explicar o elevado grau de problemas de saúde vinculados ao uso dessas substâncias. É importante que as ações de prevenção do abuso de SPAs considerem que as principais motivações para o consumo entre os jovens pesquisados estão relacionadas à busca de diversão ou de prazer. Ainda que a produção científica nacional tenha uma abordagem restrita em relação ao uso de SPAs para melhorar as notas ou o desempenho acadêmico, essa questão já se faz presente na realidade atual e, por isso, deve ser considerada com maior relevância dentro dos instrumentos de pesquisas. Novos levantamentos precisam ser realizados para permitir melhor compreensão das expectativas desse grupo, assim como auxiliar na prevenção de fatores de risco.

\section{REFERÊNCIAS}

1. Barbosa PCR, Dalgalarrondo P. O uso ritual de um alucinógeno no contexto urbano: estados alterados de consciência e efeitos em curto prazo induzidos pela primeira experiência com a ayahuasca. J Bras Psiquiatr. 2003;52:18190.

2. Barros D, Ortega F. Metilfenidato e aprimoramento cognitivo farmacológico: representações sociais de universitários. Saude Soc. 2011;20(2):350-62. http://dx.doi.org/10.1590/S0104-12902011000200008.

3. Beyer C, Staunton C, Moodley K. The implications of Methylphenidate use by healthy medical students and doctors in South Africa. BMC Med Ethics. 2014;15(1):20. http://dx.doi.org/10.1186/1472-6939-15-20. PMid:24592964.

4. Verster GC, Van Niekerk AA. Moral perspectives on stimulant use by healthy students. S Afr Med J. 2012;102(12):909-11. http://dx.doi.org/10.7196/ SAMJ.6090. PMid:23498034.

5. Maier LJ, Liechti ME, Herzig F, Schaub MP. To dope or not to dope: neuroenhancement with prescription drugs and drugs of abuse among Swiss university students. PLoS One. 2013;8(11):e77967. http://dx.doi. org/10.1371/journal.pone.0077967. PMid:24236008.

6. Duarte PCAV, Formigoni MLOS. Efeitos de substâncias psicoativas: módulo 2. 7. ed. Brasília: Secretaria Nacional de Políticas sobre Drogas; 2014. 144 p.

7. Andrade AG, Duarte PCAV, Oliveira LG. I levantamento nacional sobre uso de álcool, tabaco e outras drogas entre universitários das 27 capitais brasileiras [Internet]. Brasília: SENAD; 2010 [citado em 2016 abr 7]. Disponível em: http://www.senad.gov.br

8. Brasil. Ministério da Educação. Censo da educação superior 2014 [Internet]. Brasília; 2014 [citado em 2016 mar 5]. Disponível em: http://portal.mec. gov.br/index.php?option=com_docman\&view $=$ download\&alias $=28571$ apresentacao-censo-superior-imprensa-04-12-2015-pdf\&Itemid=30192

9. Cesar ELR, Wagner GA, Castaldelli-Maia JM, Silveira CM, Andrade AG, Oliveira LG. Uso prescrito de cloridrato de metilfenidato e correlatos entre estudantes universitários brasileiros. Rev Psiquiatr Clin. 2012;39(6):183-8. http://dx.doi.org/10.1590/S0101-60832012000600001.

10. Eckschmidt F, Andrade AG, Oliveira LG. Comparação do uso de drogas entre universitários brasileiros, norte-americanos e jovens da população geral brasileira. J Bras Psiquiatr. 2013;62(3):199-207. http://dx.doi.org/10.1590/ S0047-20852013000300004.

11. Barbosa FT. Introdução a revisão sistemática: a pesquisa do futuro [Internet]. Maceió; 2013 [citado em 2016 abr 7]. Disponível em: file:///C:/ Users/3HKPVN2/Downloads/a_introducao_a_revisao_sistematica__a_pesquisa_do_futuro.pdf

12. Sampaio RF, Mancini MC. Estudos de revisão sistemática: um guia para síntese criteriosa da evidencia científica. Rev. Bras. Fisioter. 2007;11(1):83-9. http://dx.doi.org/10.1590/S1413-35552007000100013.

13. Higgins JPT, Green S, editores. Cochrane Handbook for systematic reviews of interventions 4.2.6 [updated September 2006]. Chichester: John Wiley \& Sons; 2006

14. Menezes KKP, Avelino PR. Grupos operativos na Atenção Primária à Saúde como prática de discussão e educação: uma revisão. Cad Saude Colet. 2016;24(1):124-30. http://dx.doi.org/10.1590/1414-462X201600010162.

15. Bardin L. Análise de conteúdo: a visão de Laurence Bardin. São Paulo: Edições 70; 2011. 229 p. 
16. Cortez PA, Souza MVR, Amaral LO, Silva LCA. A saúde docente no trabalho: apontamentos a partir da literatura recente. Cad Saude Colet. 2017;25(1):113-22. http://dx.doi.org/10.1590/1414-462x201700010001.

17. Colares V, Franca C, Gonzalez E. Condutas de saúde entre universitários: diferenças entre gêneros. Cad Saude Publica. 2009;25(3):521-8. http:// dx.doi.org/10.1590/S0102-311X2009000300007. PMid:19300841.

18. Oliveira HP Jr, Brands B, Cunningham J, Strike C, Wright MGM. Percepção dos estudantes universitários sobre o consumo de drogas entre seus pares no ABC Paulista, São Paulo, Brasil. Rev. Latino-Am. Enfermagem. 2009;17(spe):871-7. http://dx.doi.org/10.1590/S0104-11692009000700018.

19. Bortoluzzi MC, Capella DL, Traebert J, Presta AA. Uso de substâncias psicoativas entre estudantes universitários em cidade do Sul do Brasil. Arq Med. 2012;26:11-7.

20. Medeiros SB, Rediess SV, Hauck N Fo, Martins MIM, Mazoni CG. Prevalência do uso de drogas entre acadêmicos de uma universidade particular do sul do Brasil. Aletheia. 2012;38:81-93.

21. Santos MVF, Pereira DS, Siqueira MM. Uso de álcool e tabaco entre estudantes de Psicologia da Universidade Federal do Espírito Santo. J Bras Psiquiatr. 2013;62(1):22-30. http://dx.doi.org/10.1590/S0047-20852013000100004.

22. Portugal FB, Cerutti C Jr, Siqueira MM. Uso de substâncias psicoativas por futuros educadores. Cad Saude Colet. 2013;21(4):432-40. http://dx.doi. org/10.1590/S1414-462X2013000400012.

23. Zalaf MRR, Fonseca RMGS. Uso problemático de álcool e outras drogas em moradia estudantil: conhecer para enfrentar. Rev. Esc. Enferm. 2009;43(1):132-8. http://dx.doi.org/10.1590/S0080-62342009000100017.

24. Gomes FC, Andrade AG, Izbicki R, Almeida AM, Oliveira LG. Religion as a protective factor against drug use among brazilian university students: a national survey. Rev Bras Psiquiatr. 2013;35(1):29-37. http://dx.doi. org/10.1016/j.rbp.2012.05.010. PMid:23567597.

25. Maia DAM, Marques RB, Maia ALM Fo. Consumo de bebidas alcoólicas e a prática do binge drinking em acadêmicos de medicina. Rev. Interdisciplinar. 2017;10(1):139-46.

26. Tostes JG, Campos FP, Pereira LGR. Consumo de álcool e outras drogas em uma Faculdade de Medicina do Sul de Minas Gerais. Rev Cienc Salud. 2016;6(2):16-24

27. Silva EC, Tucci AM. Padrão de consumo de álcool em estudantes universitários (calouros) e diferença entre os gêneros. Temas Psicol. 2016;24(1):313-23. http://dx.doi.org/10.9788/TP2016.1-21.

28. Botti NCL, Monteiro AMC, Benjamim MLN, Queiroz LC. Depression, drug addiction, ideation and suicide attempt among nursery students. Rev Enferm UFPE On Line. 2016;10(7):2611-6.

29. Silva B, Brêtas A, Pillon S, Macieira M, Carmo D, Santos M. Interface dos aspectos familiares e o uso de álcool em estudantes de enfermagem: fatores de proteção e risco. In: Atas do $5^{\circ}$ Congresso Ibero Americano em Investigação Qualitativa; 2016; Porto, Portugal. Porto: CIAIQ; 2016. p. 338-47. (vol. 2)

30. Veiga C, Cantorani JRH, Vargas LM. qualidade de vida e alcoolismo: um estudo em acadêmicos de licenciatura em educação física. Rev. Conexões. 2016;14(1):20-34.

31. Pimentel CE, Coelho LL Jr, Aragão TA. Atitudes frente ao uso de álcool, maconha e outras drogas: verificando relações de predição e mediação. Psicol Reflex Crit. 2009;22(1):29-35. http://dx.doi.org/10.1590/S010279722009000100005 .
32. Lemos K, Neves N, Kuwano A, Tedesqui G, Bitencourt A, Neves F, et al. Uso de substâncias psicoativas entre estudantes de Medicina de Salvador (BA). Rev Psiquiatr Clin. 2007;34(3):118-24. http://dx.doi.org/10.1590/ S0101-60832007000300003.

33. Carvalho AMP, Cunningham J, Strike C, Brands B, Wright MGM. Normas percebidas por estudantes universitários de três carreiras, da área da saúde, sobre o uso de drogas entre seus pares. Rev. Latino-Am. Enfermagem. 2009;17(spe):900-6. http://dx.doi.org/10.1590/S0104-11692009000700022.

34. Botti NCL, Lima AFD, Simões WMB. Uso de substâncias psicoativas entre acadêmicos de enfermagem da Universidade Católica de Minas Gerais. SMAD Rev Eletrônica Saúde Mental Álcool Drog. 2010;6(1):1-13.

35. Picolotto E, Libardoni LFC, Migott AMB, Geib LTC. Prevalência e fatores associados com o consumo de substâncias psicoativas por acadêmicos de enfermagem da Universidade de Passo Fundo. Ciênc. Saúde Colet. 2010;15(3):645-54. http://dx.doi.org/10.1590/S1413-81232010000300006.

36. Teixeira RF, Souza RS, Buaiz V, Siqueira MM. Uso de substâncias psicoativas entre estudantes de odontologia da Universidade Federal do Espírito Santo. Ciênc. Saúde Colet. 2010;15(3):655-62. http://dx.doi.org/10.1590/S141381232010000300007

37. Amorim TC, Amorim MES, Guedes LAC, Ramalho SL, Rivera GA. Evaluation of alcohol addiction among Brazilian Northeast medical students through the alcohol use disorders identification test and the relation with body mass index and smoking. Rev Bras Clin Med. 2012;10:398-401.

38. Carneiro SG, Prado AST, Moura HC, Strapasson JF, Rabelo NF, Ribeiro TT, et al. O uso não prescrito de metilfenidato entre acadêmicos de Medicina. Cad. UniFOA. 2013;1:53-9.

39. Pillon SC, O’Brien B, Piedra Chavez KA. A relação entre o uso de drogas e comportamentos de risco entre universitários brasileiros. Rev Lat Am Enfermagem. 2005;13(spe2):1169-76. http://dx.doi.org/10.1590/S010411692005000800011. PMid:16501789.

40. Morgan HL, Petry AF, Licks PAK, Ballester AO, Teixeira KN, Dumith SC. Consumo de estimulantes cerebrais por estudantes de medicina de uma universidade do extremo sul do brasil: prevalência, motivação e efeitos percebidos. Rev Bras Educ Med. 2017;41(1):102-9. http://dx.doi. org/10.1590/1981-52712015v41n1rb20160035.

41. Dázio EMR, Zago MMF, Fava SMCL. Use of alcohol and other drugs among male university students and its meanings. Rev Esc Enferm USP. 2016;50(5):785-91. http://dx.doi.org/10.1590/s0080-623420160000600011.

42. Santos HGB, Marcon SR, Espinosa MM, Baptista MN, Paulo PMC. Fatores associados à presença de ideação suicida entre universitários. Rev. LatinoAm. Enfermagem. 2017;25:e2878.

43. Damasceno RO, Boery RNSO, Ribeiro ÍJS, Anjos KF, Santos VC, Boery EN. Uso de álcool, tabaco e outras drogas e qualidade de vida de estudantes universitários. Rev. Baiana Enferm. 2016;30(3):1-10. http://dx.doi. org/10.18471/rbe.v30i3.15533.

44. Ferraz L, Rebelatto SL, Schneider GC, Anzolin V. O uso de álcool e tabaco entre acadêmicos de uma universidade do sul do Brasil. Rev. Bras. Promoc. Saúde. 2017;30:79-85. http://dx.doi.org/10.5020/18061230.2017.p79.

45. Petroianu A, Reis DC, Cunha BD, Souza DM. Prevalência do consumo de álcool, tabaco e entorpecentes por estudantes de medicina da Universidade Federal de Minas Gerais. Rev Assoc Med Bras. 2010;56(5):568-71. http:// dx.doi.org/10.1590/S0104-42302010000500019. PMid:21152830.

46. Oliveira MG. Desenvolvimento, aprimoramento e consolidação de uma educação nacional de qualidade [Internet]. Brasília: Conselho 
Nacional de Educação Conselho Pleno; 2013 [citado em 2016 abr 10]. Disponível em: http://portal.mec.gov.br/index.php?option=com docman\&view=download\&alias $=15885$-educacao-profissional-tecnologicaproduto-1-pdf\&category_slug=junho-2014-pdf\&Itemid $=30192$

47. Cruz MS, Felicissimo M. Problemas médicos, psicológicos e sociais associados ao uso abusivo de álcool e outras drogas. In: Duarte PCAV, Formigoni MLOS, editores. Efeitos de substâncias psicoativas: módulo 2. 7. ed. Brasília: Secretaria Nacional de Políticas sobre Drogas; 2014. 144 p.

48. Maier LJ, Liechti ME, Herzig F, Schaub MP. To dope or not to dope: neuroenhancement with prescription drugs and drugs of abuse among
Swiss university students. PLoS One. 2013;8(11):e77967. http://dx.doi. org/10.1371/journal.pone.0077967. PMid:24236008.

49. Brandt SA, Taverna EC, Hallock RM. A survey of nonmedical use of tranquilizers, stimulants, and pain relievers among college students: patterns of use among users and factors related to abstinence in nonusers. Drug Alcohol Depend. 2014;143:272-6. http://dx.doi.org/10.1016/j. drugalcdep.2014.07.034. PMid:25150402.

Recebido em: Jun. 25, 2017

Aprovado em: Jan. 19, 2018 